\title{
The Classroom Attendance Management System of Face Recognition Based on LBS
}

\author{
Fengping Cao ${ }^{a}$, Mimi Wang ${ }^{b}$, and Kuihao Wang ${ }^{c}$ \\ Southeast University Chengxian College, Nanjing, 210088, China. \\ acfp423@126.com, ${ }^{\text {byimi0516@163.com, }{ }^{c} 1051992187 @ q q . c o m}$
}

Keywords: Student attendance, face recognition, APP, LBS

\begin{abstract}
Face recognition is a popular biometric technology, it is based on facial features, face image recognition to identify the identity of the corresponding person. Face identity identification, has been widely used in banking, transportation, company attendance and other fields. This paper puts forward a face recognition system based on LBS, in which students use the mobile terminal face recognition function to complete the classroom attendance independently, and the data of the mobile terminal and the face recognition are compared to the server data to generate the attendance. The system effectively avoids manual attendance efficiency low, cheating on Attendance, the error rate is high, cannot do to the study process comprehensive supervision, cannot form the statistical data and so on.
\end{abstract}

\section{Introduction}

With the urgent demands of society for fast and effective authentication, biometric features become the most ideal basis for automatic authentication. As a kind of biometric recognition [1], face recognition is a popular biometric recognition technology, which is based on facial features and recognition of face image, so as to realize identity identification. Compared with recognition methods such as fingerprint identification, retinal recognition, iris recognition and gait recognition, face recognition has the characteristics of directness, friendliness and convenience, thus obtaining extensive research and application [2-3].This paper presents a face recognition system based on LBS for the classroom attendance in classroom, the use of Baidu LBS and Baidu's face recognition service of these two third-party services to achieve a more accurate class attendance, Only attendance occurs within a certain period of time, longitude, latitude range, as effective. It avoids the drawbacks of other automatic attendance, such as: Scanning course two-dimensional code attendance mode mobile terminal in other places outside the classroom, the external scanning course of two-dimensional code cheating attendance, classroom camera face recognition attendance, the classroom dim light Causes facial features are not obvious, attendance success rate is low [4].

LBS(Location Based Service ) refers to providing location-based services for users under mobile computing conditions through space positioning technology, geo-information technology, embedded technology and wireless network technology [5]. Baidu LBS to provide intelligent positioning services, relying on Baidu's powerful location data information. Combined with GPS, WFFI, base station information is positioned. IP positioning can be controlled within a range of dozens of meters. Baidu's face recognition service, based on in-depth learning of face recognition scheme, accurate recognition of the face information in the picture, providing human face attribute recognition, key point positioning, face 1:1 ratio, face 1:n recognition, live detection and other capabilities, its face recognition technology international leader, recognition accuracy rate of more than $99 \%[6]$

\section{The Proposed Model of Classroom Attendance}

The system Mobile Terminal adopts C/S architecture, and the Web server adopts B/S architecture. Systematic research and design for the purpose of providing technical support for 
classroom attendance of Chengxian College of Southeast University. In the whole system design process analysis of Southeast University Chengxian College actual data needs, such as Student's class time, teaching building longitude, latitude, leave information and so on. The system includes mobile clients and servers. Students use mobile terminals to complete the classroom face recognition real-time attendance, request leave and leave the certificate upload process. Teachers to achieve classroom attendance management, review the Leave list, check attendance information, find the students who are near the limit of their maximum absence allowance. The following figure 1 shows the system structure.

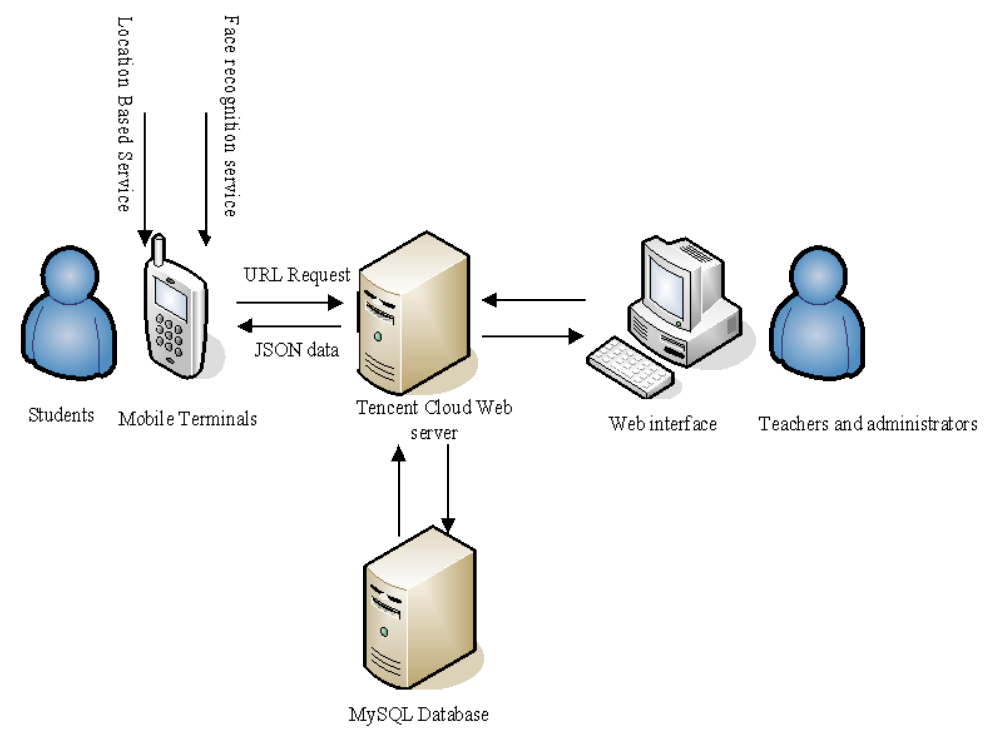

Fig. 1 System structure

The teacher uses the Web service side to confirm course details before class. Students log in on the APP side and complete face recognition attendance within the time specified by the teacher. The server compares the human face information, location information and attendance time information of the mobile terminal with the data of the database, and determines whether the students ' attendance is successful and writes the attendance results to the database. Students who do not complete attendance at 5 minutes after class are considered absent. Students can use the APP to select the relevant course, fill out the relevant leave information, complete the leave operation, the teacher on the server to complete the Leave review.

\subsection{Process of Attendance.}

Download Attendance App to mobile terminals, the first time to fill in the name, school number, photos and other registration information to complete registration, before class, using registration information login to the APP to complete face recognition attendance. Before the student attendance, first select Attendance course information, click the Start Attendance button, the system starts the camera to face recognition, the server will be the mobile end of the attendance data and database to match, in attendance position, attendance time, face recognition are matched when the attendance is successful, otherwise attendance failure. When the attendance is completed, the system will write the attendance information back to the system database, and the students can query the attendance result information after refreshing. Figure 2 below shows the student attendance process.

The teacher logs on to the Web server side to see the attendance information for the course. Attendance information According to the teacher's class information in tabular form, absence with "0" said, sign in successfully with "1" said, late with "-" said, leave with "+" said. The form data is updated in real time according to the attendance information of the students, and the teacher can check the history attendance statistics of the course, and check the student information of the high absence frequency according to the number of absences set. 


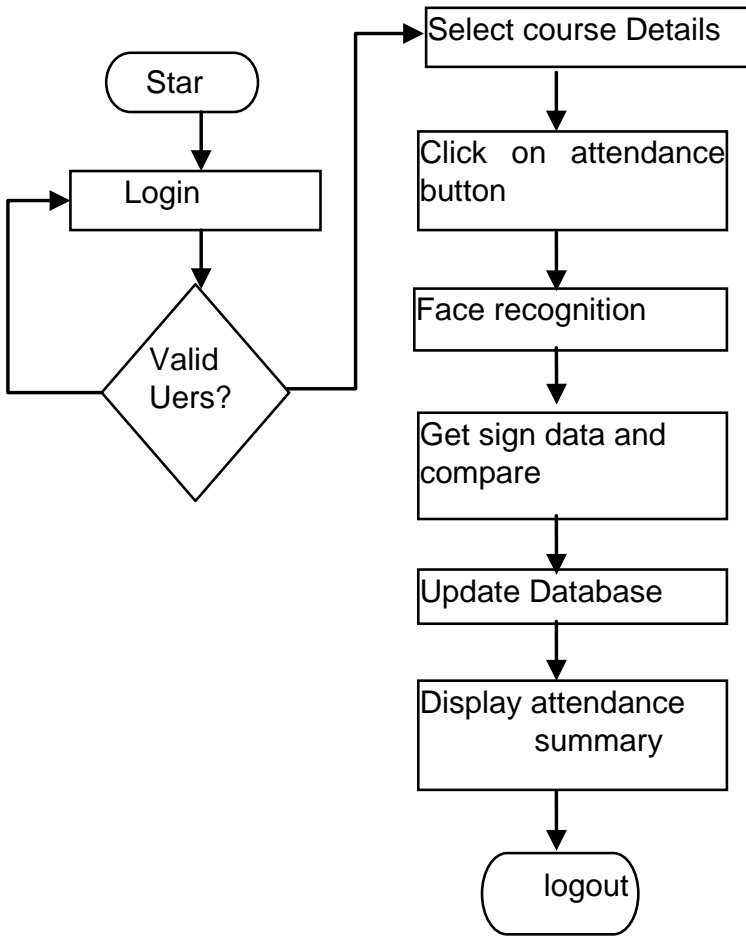

Fig.2 The process of student attendance

\subsection{Database Schema}

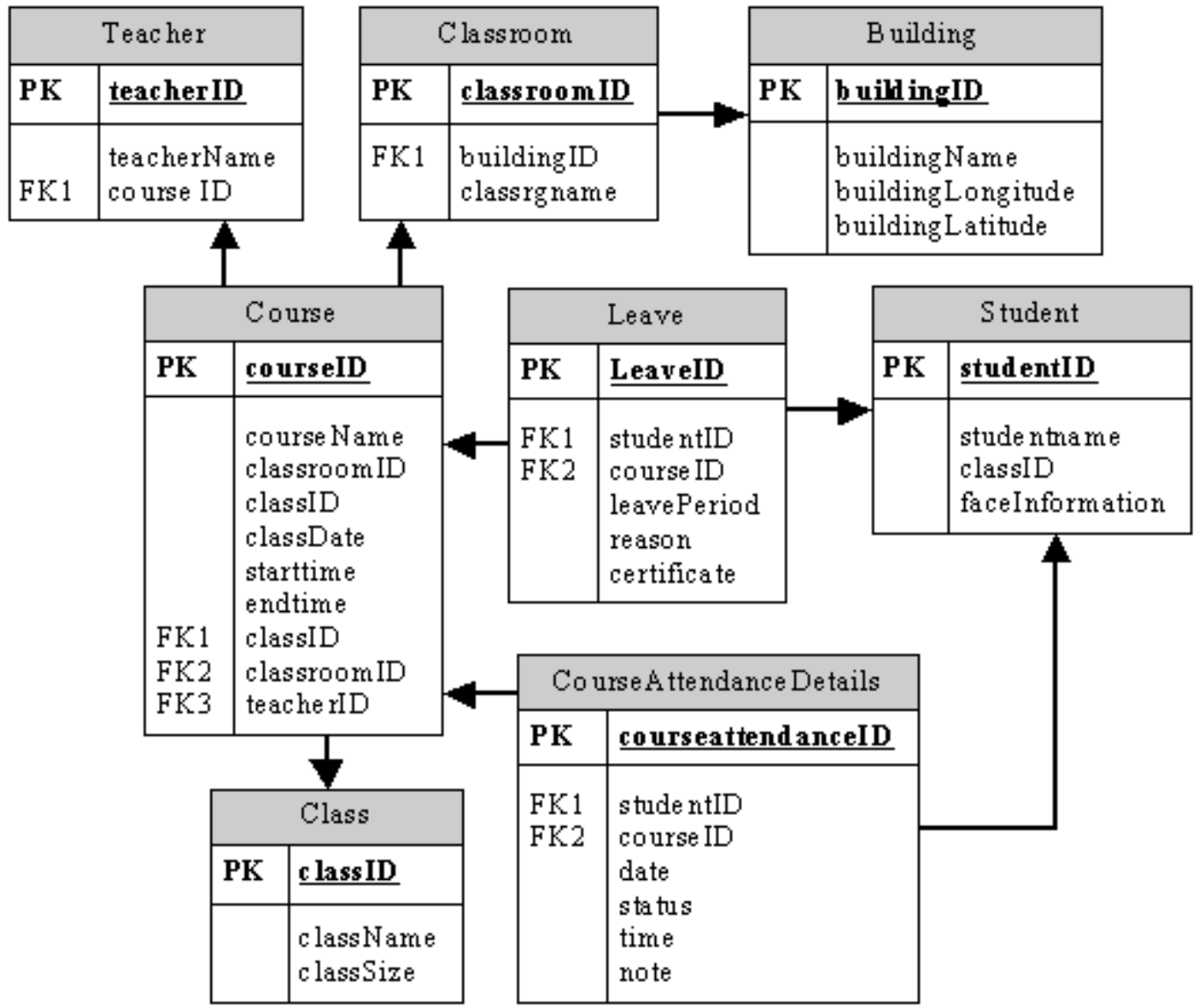

Fig.3 Database schema

A large amount of time and focus on the design of a stable and reliable database. The database schema is depicted below Figure 3. It shows what will be necessary for the system in order to record all attendances of each student. 
As can be seen above the database will consist of eight tables:

- Building (contains the building ID of the building and is also the primary key, building name, building longitude and building latitude on the particular building)

- Class (class ID as primary key, class name and class size on the class )

- Course (course ID which is the primary key, course name, classroom ID, class ID, teacher ID, class date, start time and end time on the particular course)

- Classroom (classroom ID as primary key, building ID and classroom name on the particular Classroom)

- CourseAttendanceDetails (courseAttendanceID which is the primary key, student ID, course ID, date, status, time and note on the particular details of attendance)

- Student(student ID as primary key, student name, class ID and face information as his/her personal info)

- Teacher (Teacher ID as primary key, teacher name)

- Leave (Leave ID as primary key, student ID, course ID, Period, reason and certificate on the particular details of Leave)

\section{Prototype System Application}

The system uses the Tencent cloud server as the shared cloud virtual host, Nginx1.10.2 as the server, MySQL as the database management system. Use Andriod Studio to implement front-end functionality development. Using the PHP language, the Laravel framework, Laravel-admin completes the backend application development. Baidu Echarts Data Visualization Chart Library to complete the rich and diverse background data analysis and statistics.

The system is run in classrooms with wireless network coverage, The system is divided into Web server and mobile terminal app. The student installs the system mobile app on the mobile terminal that has the camera function, the teacher uses the computer to operate the Web server. Server operating Environment: Intel skylake Xeon Platinum 8163 2.5GHz processor, 8G memory, 2.5GHZ processor frequency, 1Gbps intranet bandwidth, 300,000 PPS intranet packet. Ubuntu 16.04 operating system, 64-bit .Android platform environment: Xiao Quad-core 2.5GHz processor, 2GB memory, 16GB high speed flash, Mobile Terminal environment: Android more than 4.0.

\subsection{Android Client.}

The mobile client mainly completes the information management and the classroom attendance function, the information management includes the user Information update, the Personal day attendance information and this month attendance information statistics and the inquiry, as shown in Figure 4.

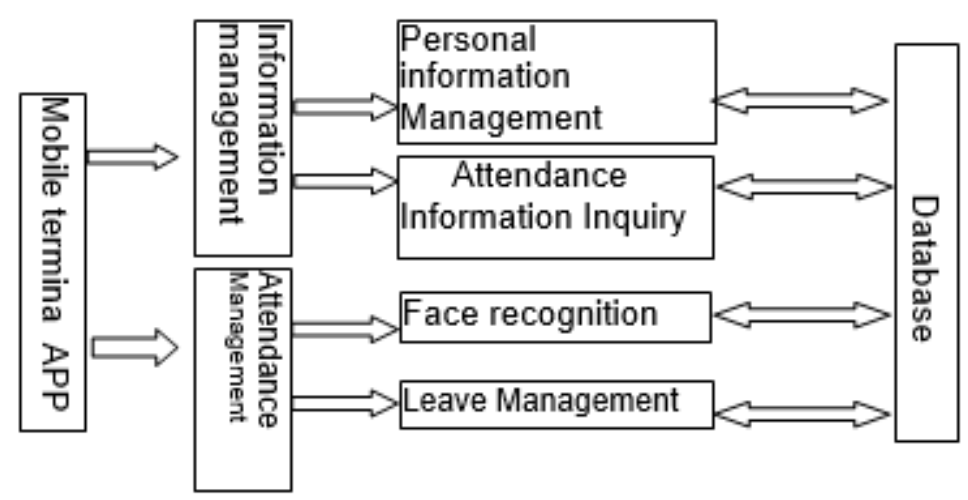

Fig.4 Mobile client function module

\subsection{Web Services.}

The functionality of the Web service is shown in Figure 5. Basic information maintenance, completion of students, courses, teachers basic information maintenance, attendance information 
inquiries, check the historical attendance data, attendance data summarized in the near period of time students attendance, leave, absence details, Figure 7 shows the recent period of time attendance statistics; Leave management, the teacher may complete the leave review according to the student's request for leave certificate.

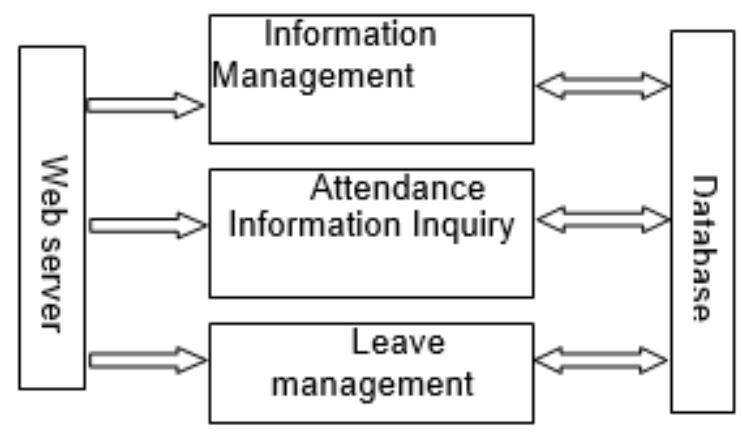

Fig.5 Web service function module

\section{Operation Result Analysis}

System in the Southeast University Chengxian College in part of the classroom attendance of the application debugging, and the attendance results data for detailed analysis.

\subsection{Mobile terminal attendance data analysis}

Students login to the system, within the stipulated time (usually within 5 minutes after class) to select the course of the day curriculum, the completion of face recognition attendance, class 5 minutes after the face recognition attendance will be identified as late. No face recognition processes and face recognition absences that are not in the specified longitude or latitude range are identified as absence. The mobile end can display the attendance date, course, attendance status and memo information in detail. Table 1 Details of part of the attendance test records that a student has read from a mobile terminal. student in the software engineering, operating system principles corresponding to the classroom, in the course of 5 minutes before and after the completion of the face recognition attendance, attendance status of "1" (attendance); student in the network and information security courses in the classroom completed the face attendance, because in the class after 20 minutes, so identified as "-" (late) .student although in the stipulated time to complete the face recognition attendance, but because the position is not in the multimedia technology curriculum corresponding classroom so is regarded as "0" (absence); a classmate in the Linux application Development course submitted a leave form, the teacher has not yet approved, so show pending approval.

The test shows that the mobile end can complete the face recognition attendance and display the attendance status accurately.

Table 1 Mobile terminal attendance data

\begin{tabular}{cccc}
\hline Date & Course & $\begin{array}{c}\text { Attendance } \\
\text { Status }\end{array}$ & Notes \\
\hline 2018-05-07 & Software Engineering & 1 & $8: 03$ \\
2018-05-07 & Software Engineering & 1 & $9: 54$ \\
2018-05-07 & Network and information & - & $14: 00$ \\
& security & & \\
2018-05-08 & Multimedia Technology & 0 & $8: 01$ \\
$2018-05-08$ & Linux Application & + & $\begin{array}{c}\text { Waiting for } \\
\text { review }\end{array}$ \\
\hline
\end{tabular}

\subsection{Summary analysis of Attendance}

The teacher logs on to the Web server side, queries the attendance data, and notes the absence 
document, and the absence document is identified as absent. Due to the large number of web-side check-in data, here is a course to select one day part of the attendance data for analysis.

As shown in Table 2, the attendance time and attendance status of the first two students are accurately displayed, the third student is late, the fourth student is absent and the fifth student is absence.

Table 2 Summary of course attendance

\begin{tabular}{cccc}
\hline $\begin{array}{c}\text { Student } \\
\text { Number }\end{array}$ & Status & $\begin{array}{c}\text { Attendance } \\
\text { time }\end{array}$ & Leave list \\
\hline 38115301 & 1 & $8: 03$ & \\
38115302 & 1 & $8: 01$ & \\
38115303 & - & $8: 16$ & \\
38115304 & + & & review button \\
38115305 & 0 & & \\
\hline
\end{tabular}

Experimental results show that the Baidu LBS (Location Based Services) interface of highprecision, efficient positioning function, can adapt to indoor positioning needs, to determine the location of classroom attendance; Baidu face recognition, high-precision, With high accuracy, face recognition and face alignment are realized, which ensures the authenticity of attendance. The leave module facilitates students to submit applications for leave online. The system can basically meet the requirements of classroom attendance.

\section{Summary}

The system adopts Tencent Cloud server, designs and realizes the classroom attendance system based on the Baidu LBS (Location Based Services) and the Baidu face recognition, the system includes the mobile client and the Web server side. The students can implement the classroom attendance, historical attendance query and leave operation according to the face recognition and positioning system, and the teacher realizes basic information maintenance, attendance Information inquiry and leave management on the web side. The system has rich chart statistic function, the visual display student Attendance detail, facilitates the university classroom data analysis.

Through the System application test, the system can effectively improve the classroom attendance management efficiency, effectively solve the manual call attendance and statistical inefficiency of the problem, accurate rate of nearly 99\%. In the next future there can be also other improvements to the system where the system can send SMS to the student who will know immediately that her/his absence has been recorded.

\section{References}

[1] Biometrics Market and Industry 2007-2012[R].International Biometric Group, 2007.

[2] Song Jiacheng. The status and development of face recognition technology [J]. Electronic technology and software engineering, 2017, (17): 104

[3] Wu Meixiang, Deng Yuanyuan, Pei Fenghua, et. Design and implementation of a mobile classroom attendance system based on face recognition [J].Software, 2018 (1): 5-8.

[4] Liu Yang. Design and implementation of classroom attendance system based on face recognition technology [D]. Southwest Jiaotong University, 2016.

[5] Chen Fly, Yang Chongjun, Shen shengli, etc. research on mobile GIS based on lbs [J]. Computer Engineering and Applications, 2006, 42 (2): 200-202.

[6] Official website: https://cloud.baidu.com/product/face/compare 